\title{
Examining the theoretical relationships between constructs in the person-centred practice framework: a structural equation model.
}

\section{Tanya McCance}

Ulster University School of Nursing

Brendan McCormack

Queen Margaret University Division of Nursing Edinburg

\section{Paul Slater ( $\nabla$ pf.slater@ulster.ac.uk)}

University of Ulster at Jordanstown https://orcid.org/0000-0003-2318-0705

\section{Donna McConnell}

Ulster University School of Nursing

\section{Research article}

Keywords: Person-centred practice, structural equation model, cross sectional survey

Posted Date: May 3rd, 2020

DOl: https://doi.org/10.21203/rs.3.rs-22586/v1

License: (-) This work is licensed under a Creative Commons Attribution 4.0 International License. Read Full License

Version of Record: A version of this preprint was published at International Journal of Environmental Research and Public Health on December 13th, 2021. See the published version at https://doi.org/10.3390/ijerph182413138. 


\section{Abstract}

Background Research relating to Person-centred practice continues to expand and currently there is a dearth of statistical evidence that tests the validity of an accepted model of person-centred practice. The Person-centred Practice Framework is a leading model of practice and this study aims to examine it statistically.

Methods A cross sectional survey design using a standardized tool was used to assess a purposive sample $(n=1283,31.8 \%)$ of multi-disciplinary health professionals across both Northern and Southern Ireland. Survey items scores were summed to provide construct scores and included in a structural model to examine the theoretical model of person-centred practice. Full ethical approval was gained.

Results The results were drawn from a multi-disciplinary sample, and representing a broad range of clinical settings. The model explains $60.5 \%$ of the total variance. Factor loadings on to the second order latent construct, and fit statistics confirm the acceptability of the measurement model. Statistically significant factor loadings were acceptable. A positive and statistically significant relationship was observed between prerequisites and care environment as well as care processes.

Conclusions The study provides statistical evidence to support the Person-centred Practice Framework, with a multidisciplinary sample. The findings help confirm the effectiveness of the PCPI-S and an instrument to measure an internationally renowned model of Person-centred practice.

\section{Introduction}

The value placed on person-centredness as the preferred approach in health and social care is uncontested, as evidenced in policy and strategy development globally $[1,2,3]$. The knowledge base underpinning person-centredness continues to expand, with greater clarity on person-centredness as a concept relevant to international healthcare, and an increased understanding of the key components that need to be considered for effective implementation of person-centred practice $[4,5,6]$. This has led to the development of conceptual frameworks and models depicting the components of person-centredness (e.g. [7, 8]), alongside the development of tools to enable measurement [9]. This paper presents the outcomes from a programme of work that has focused on the development of a conceptual framework for implementing person-centred practice [10,11], and development of a tool aligned to the framework that can offer a valid and reliable standardised measure [12].

\section{Background}

It is generally accepted that the principles underpinning person-centredness as an approach focuses on: treating people as individuals; respecting their rights as a person; building mutual trust and understanding; and developing positive relationships. Furthermore, these principles reflect a standard of care that practitioners aspire to in their professional practice. The challenge, however, continues to be how these principles are translated into everyday practice to enable multiprofessional teams to deliver this standard of care consistently over time $[13,14]$.

The Person-centred Practice Framework (PCPF) was originally born out of a desire to operationalise person-centredness in a way that would illuminate practice, and provide practitioners with a language that would enable them to name components of personcentredness and the barriers and enablers that influence its development in the workplace.

The PCPF comprises four domains which are briefly described below.

1. Prerequisites, which focus on the attributes of the nurse and include: professionally competent, developed interpersonal skills, commitment to the job, clarity of beliefs and values, and knowing self.

2. Care environment, which focuses on the context in which care is delivered and includes: appropriate skill mix; shared decision-making systems; effective staff relationships; supportive organisational systems; power sharing; potential for 
innovation and risk-taking; and the physical environment.

3. Person-centred processes, which focus on delivering care to the patient through a range of activities and include: working with patient's beliefs and values; being engaged, having sympathetic presence, sharing decision-making and providing holistic care.

4. Outcomes, which are the results of effective person-centred practice and include: experience of good care; involvement with care; feeling of well-being; and existence of a healthful culture.

In the most recent version of the PCPF an additional element has been added to take account of the macro context. This reflects the factors that are strategic and political in nature that influence the development of person-centred cultures and include: health and social care policy; strategic frameworks; workforce developments; and strategic leadership.

The relationship between the four main constructs of the PCPF is represented pictorially, that being, to reach the centre of the framework, the attributes of staff must first be considered, as a prerequisite to managing the care environment, in order to provide effective care through the care processes. This ordering, ultimately leads to the achievement of the outcomes - the central component of the framework (refer to Figure 1). It is also acknowledged that there are relationships between the constructs. The original Person-centred Nursing Framework was described as a mid-range theory [15]. It's place on the continuum of theory development was made explicit by McCormack \& McCance [10,11] drawing on the seminal work of Fawcett [16]. Fawcett argues that mid-range theories articulate one or more relatively concrete and specific concepts that are derived from a conceptual model. Furthermore, the propositions that describe these concepts propose specific relationships between them, and empirical indicators provide the means of measuring concepts within a mid-range theory. The Person-Centred Practice Framework meets the criteria for a mid-range theory, in that it has been derived from two abstract conceptual frameworks $[17,18]$, comprises concepts that are relatively specific, and outlines relationships between the concepts.

\section{Please insert Figure 1 here.}

Furthermore, recent advancements have been made to develop empirical indicators to measure concepts within the PCPF. The development of the Person-centred Practice Inventory - Staff (PCPI-S) provides a measure that effectively maps directly onto the constructs of the PCPF. De Silva [9] conducted a review of existing tools to measure person-centred practice and reported that of the 114 instruments identified, none provided direct measurement of the concepts of person-centred practice. Many quantitative tools relied on statistical techniques that draw heavily on a posteriori classification of constructs that emerge from reductionist techniques such as exploratory factor analysis.

The PCPI-S holds to the concept of theory driven instrument development [12]. Instrument items map directly to accepted definitions that make up the model: factors onto items and second order latent variables onto first order factors. It provides quantitative data to allow testing of the theoretical model that underpins it. The comprehensiveness of the PCPF and as a consequence its complexity, gives the model an advantage over other simplistic models that reduce person-centred practice to measurements of broad, ill-defined concepts. These use, at best, proxy measures and add little to advance understanding of how to effectively measure and move toward person centred outcomes.

\section{Methods}

A quantitative cross sectional survey research design using the PCPI-S was used to generate sufficient data to adequately test the measurement model. The PCPI-S is a 59-itemed instrument that measures 17 constructs aligned to the Person-centred Practice Framework [11]. All items of the PCPI-S are measured on a five point scoring range (1-Strongly Disagree to 5 - Strongly Agree), 
with higher scores indicating higher levels of agreement. Demographic details of participants were also collected. Factor structure and psychometric properties of the PCPI-S are reported by Slater et al [12] and Bing-Jonsson et al [19] and are acceptable.

\section{Sample}

The PCPI-S was tested with a sample of health professionals drawn from 6 organisations representing 14 acute hospital settings and 45 clinical units in Northern Ireland and Republic of Ireland. Organisations, hospital settings and clinical sites were all selfnominating to participate in the various studies, using the PCPI-S, to examine the current state of person-centred practice in Ireland. A range of clinical services were invited to participate in the overall study, including: Adult Services; Children and Young People; Primary Care and Older People; Mental Health, Emergency Department, Learning Disability, and Maternity. The settings were selected so as to obtain a good representation of views on person-centred practice for health professionals across the participating organisations. A gatekeeper in each clinical unit randomly distributed questionnaire packs to all health professionals working within the unit. The following inclusion criteria was applied: (i) working full time' (ii) have worked in the clinical setting for at least 6 months; and (iii) willing to participate.

\section{Ethical Approval}

Full ethical approval was sought and gained from the relevant organisational and ethical bodies in line with research governance requirements. The main ethical issues related to informed consent and assuring confidentiality and anonymity for all participants.

\section{Procedure}

Questionnaires were distributed to the gatekeeper in each clinical setting with the consent of the unit nurse manager/clinical nurse manager who provided a list of the total population of health professionals in the settings. A total of 4039 questionnaire packs were distributed across clinical settings. Participants were asked to complete the questionnaire and return it in the envelope provided for collection by a researcher (Data collected Mar - Dec 2015). The process of implied consent was made explicit in the Participant Information Sheet and sought whereby a completed questionnaire implies consent to participate. This ensured the confidentiality and anonymity of returned questionnaires. A deadline of two weeks was given for the return of questionnaires and a week of follow-up visits to retrieve them. Questionnaires collected were collated and categorised for data analysis by construct and clinical setting. A response rate of $31.8 \%(n=1283)$ was achieved (refer to Table 1$)$.

\section{Measurement Model}

Construction of the measurement model for testing was based on the PCPF (refer to Figure 1). The 59 items of the PCPI-S map onto the 17 constructs of the framework, and the constructs onto second order latent factors. The relationship between the items and their unobserved first order latent variables assumes the first order latent variables drives the indicators (i.e. these indicators are assumed to be observable instances or manifestations of their unobserved latent variables, and thus changes in the unobserved latent variables are 'indicated' by observable changes in the items in its measure [20]. The relationship between item and first order unobserved latent variable psychometric properties has been previously presented [12, 13].

Similarly, a second order latent variable assumes the first order latent variables to be 'driven' by the second order latent variable and its subsequent filter down to the items. Wang and Wang [20] suggest that the aggregation of items, to first order and 
eventually to broad second order latent variables allows for complex models to be simplified and interaction effects between the second order variables examined. In this paper, structural modelling using actual scores for each construct will be used to fit the second order latent variable measurement model and the relationship between the second order variables. The PCPF model postulates a direct relationship between second order latent variables Prerequisites and the Care Environment and subsequently Care Processes. This measurement model (see Figure 2) was tested as the initial model. Given the relative newness of the PCPI-S and the lack of comparative findings relating to the testing and fitting of the PCPF, a pragmatic approach to the specification of the model was applied.

\section{Statistical Analysis}

The data were prepared in line with descriptive and measures of dispersion statistics were generated for all items to help inform subsequent analysis. Inter-item correlations were generated to examine for collinearity prior to full analysis. Confirmatory factor analysis was used to examine the theoretical measurement model. Examination of the data indicates skewness and kurtosis on many of the items. Therefore, the data were analysed using Maximum Likelihood Robust (MLR) as relevant with continuous and skewed data.

The model was re-specified using the modification indices provided in the statistical output until acceptable and a statistically significant relationship identified. All re-specifications of the model were guided by principles of meaningfulness (a clear theoretical rationale); Transitivity (if A is correlated to B, and B correlated to C, then A should correlate with C); Generality rule if there is a reason for correlating the errors between one pair of errors, then all pairs for which that reason applies should also be correlated [21].

Acceptance modification criteria included the following:

1. within factors inter-item correlated errors were permitted and based on criteria of being theoretically relevant introduced one at a time and selected on highest score first (exceeding scores of 3.98).

2. across factors inter-item correlated errors were permitted and based on criteria of being theoretically relevant introduced one at a time and selected on highest score first (exceeding scores of 3.98)

3. only statistically significant relationship retained to help produce as parsimonious a model as possible.

Acceptable fit statistics were set at Root Mean Square Estimations of Approximation (RMSEA) of 0.08 or below; $90 \%$ RMSEA higher bracket below 0.08; and Comparative Fit Indices (CFI) of 0.90 or higher and TFI below 0.05 [20, 22]. Hair et al [23] advocate a factor loading of 0.30 for a sample size of greater than $n=350$.

\section{Results}

A breakdown of the demographic details is outlined in Table 1. There was a good spread of responses across banding and experience. There was an uneven distribution across healthcare setting in the total sample as the organisations requested different samples to be surveyed. 


\begin{tabular}{llllll} 
Profession & \multicolumn{3}{c}{ Experience } & \multicolumn{2}{l}{ Nurses Only } \\
\hline Nursing & $84.4 \%(n=1088)$ & $<1$ year & $3.6 \%(n=46)$ & Band 5 & $64.1 \%(431)$ \\
\hline Medical & $6.7 \%(n=86)$ & $1-5$ years & $24.1 \%(n=305)$ & Band 6 & $20.1 \%(135)$ \\
\hline AHP & $7.0 \%(n=90)$ & $6-10$ years & $15.7 \%(n=199)$ & Band 7 & $14.6 \%(98)$ \\
\hline HCA & $1.9 \%(n=25)$ & Over 10 years & $565 \%(n=715)$ & Band 8 & $1.2 \%(8)$
\end{tabular}

Table 1. Demographic spread of overall sample

All 17 constructs were positively scored at 'Agreed' (Table 2). The lowest scored construct was 'Shared Decision Making Systems' and the highest was 'Providing Holistic Care'. Measures of skewness and kurtosis were acceptable and indicate a normality of distribution.

Table 2. Mean scores and measures of distribution for constructs 


\begin{tabular}{lccccc} 
CONSTRUCTS & Mean & SD & Skewness & Kurtosis & \\
\hline PREREQUISITES & 4.16 & .37 & -.462 & 1.89 & .77 \\
\hline Professionally Competent & 4.24 & .46 & -.498 & 1.549 & .48 \\
\hline Developed Interpersonal Skills & 4.32 & .43 & -.337 & .755 & .67 \\
\hline Being committed to the job & 4.39 & .47 & -.749 & .747 & .74 \\
\hline Knowing Self & 3.96 & .58 & -.766 & 1.316 & .63 \\
\hline Clarity of beliefs and values & 3.90 & .58 & -.849 & 2.692 & .62 \\
\hline THE CARE ENVIRONMENT & 3.76 & .51 & -.698 & 1.18 & .75 \\
\hline Skill mix & 4.15 & .51 & -.651 & 1.505 & .40 \\
\hline Shared decision making systems & 3.55 & .78 & -.608 & .264 & .75 \\
\hline Effective staff relationships & 3.94 & .76 & -1.148 & 1.803 & .83 \\
\hline Power sharing & 3.78 & .74 & -.888 & 1.086 & .79 \\
\hline Potential for innovation and risk taking & 3.80 & .67 & -.569 & .953 & .87 \\
\hline The Physical Environment & 3.96 & .60 & -.721 & 1.500 & .84 \\
\hline Supportive Organisation Systems & 3.18 & .83 & -.464 & -.125 & .86 \\
\hline CARE PROCESSES & 4.18 & .44 & -.325 & 1.41 & .88 \\
\hline Working with patients beliefs and values & 4.06 & .56 & -.459 & 1.479 & .78 \\
\hline Shared Decision making & 4.09 & .58 & -.430 & .880 & .74 \\
\hline Engagement. & 4.20 & .47 & -.210 & 2.509 & .78 \\
\hline Sympathetic Presence & 4.21 & .51 & -.526 & 1.634 & .70 \\
\hline Providing holistic care & 4.30 & .53 & -.571 & .622 & .78 \\
\hline & & & & & \\
\hline
\end{tabular}

The correlation matrix scores (see table 3) indicate a positive correlation between the 17 measures at a low to moderate size. There were no issues of collinearity between construct scores.

Table 3. Correlation matrix of Constructs of PCPI-S 


\begin{tabular}{|c|c|c|c|c|c|c|c|c|c|c|c|c|c|c|c|c|c|}
\hline & 1 & 2 & 3 & 4 & 5 & 6 & 7 & 8 & 9 & 10 & 11 & 12 & 13 & 14 & 15 & 16 & 17 \\
\hline V1 & 1 & & & & & & & & & & & & & & & & \\
\hline V2 & 543 & 1 & & & & & & & & & & & & & & & \\
\hline V3 & 557 & 570 & 1 & & & & & & & & & & & & & & \\
\hline V4 & 377 & 441 & 438 & 1 & & & & & & & & & & & & & \\
\hline V5 & 321 & 300 & 345 & 380 & 1 & & & & & & & & & & & & \\
\hline V6 & 357 & 410 & 355 & 315 & 427 & 1 & & & & & & & & & & & \\
\hline V7 & 378 & 329 & 335 & 304 & 446 & 454 & 1 & & & & & & & & & & \\
\hline V8 & 287 & 323 & 264 & 225 & 314 & 343 & 570 & 1 & & & & & & & & & \\
\hline V9 & 365 & 351 & 299 & 278 & 305 & 378 & 626 & 744 & 1 & & & & & & & & \\
\hline V10 & 365 & 322 & 298 & 286 & 344 & 339 & 401 & 354 & 457 & 1 & & & & & & & \\
\hline V11 & 385 & 380 & 457 & 355 & 359 & 355 & 386 & 330 & 397 & 341 & 1 & & & & & & \\
\hline V12 & 250 & 246 & 249 & 309 & 268 & 285 & 516 & 566 & 666 & 347 & 387 & 1 & & & & & \\
\hline V13 & 517 & 463 & 520 & 422 & 344 & 351 & 340 & 332 & 418 & 382 & 469 & 368 & 1 & & & & \\
\hline V14 & 438 & 447 & 465 & 339 & 307 & 347 & 337 & 340 & 391 & 293 & 387 & 337 & 601 & 1 & & & \\
\hline V15 & 448 & 505 & 466 & 337 & 292 & 373 & 260 & 229 & 296 & 315 & 347 & 212 & 552 & 655 & 1 & & \\
\hline V16 & 570 & 566 & 565 & 399 & 330 & 352 & 320 & 300 & 352 & 342 & 383 & 282 & 571 & 590 & 618 & 1 & \\
\hline V17 & 456 & 480 & 555 & 380 & 259 & .321 & 310 & 336 & 396 & 292 & 477 & 313 & 696 & 560 & 516 & 612 & 1 \\
\hline
\end{tabular}

\section{Theoretical Framework}

The Kaiser-Meyer-Olkin Measures of sampling adequacy 0.932; Bartletts test of sphericity (chi square $=11.41, d f=136, p=0.001$ ). KMO scores of $\geq 0.8$ and Bartletts test of significant $p \geq 0.05$ are acceptable values. The original model failed to have acceptable fit statistics (see table 4) and modifications to the original model were required.

Table 4. Fit Statistics for alternative measurement models of the PCPI-S

\begin{tabular}{llllll} 
Model & RMSEA & $90 \%$ RMSEA & CFI & TLI & SRMR \\
\hline Original Model & 0.079 & $0.075-0.083$ & 0.889 & 0.870 & 0.076 \\
\hline Accepted Model & 0.034 & $0.033-0.035$ & 0.901 & 0.893 & 0.049
\end{tabular}

\section{Correlated Error of Variances}

A series of correlated errors were introduced to the model in an iterative process using the criteria outlined. This process continued to a position where the fit statistics were acceptable. The presence of correlated errors within factors indicates the principle of Transitivity; generality and meaningfulness. There were 5 correlated error variance corrections included in the model. The 5 correlated errors of variance indicate a presence of an unmeasured source of influence (generality) and/or may be accounted from with justification of the relative closeness in the factors in the model (Meaningfulness). The issue of generality does indicate an incomplete measurement model and that the questionnaire would be improved with the inclusion of factors that may not be adequately addressed in this model. The process of instrument modification resulted in acceptable fit statistics. 
A three factor model explains $60.47 \%$ of the variance. All constructs were statistically significant. Examination of the Cronbach's alpha scores indicate acceptable factor structures: Prerequisites Cronbach's alpha $=0.77$; Care Environment $=0.75$; and Care Processes $=0.88$ and are acceptable. Examination of the factor loadings show the constructs 'Developed Interpersonal Skills' and 'Being committed to the job' offer the largest contribution to 'Prerequisite' and 'Clarity of beliefs and values' as least significant. 'Shared Decision-making' was the most significant predictor of 'Care environment' and all seven constructs contributed significantly to Care environment. All five constructs contributed significantly to 'Care Processes' but 'Sympathetic Presence' was the main contributor.

\begin{tabular}{|c|c|c|c|c|}
\hline CONSTRUCTS & Estimate & S.E. & Est./S.E. & Variance \\
\hline \multicolumn{5}{|l|}{ PREREQUISITES } \\
\hline Professionally Competent & 0.727 & 0.020 & 36.73 & $47 \%$ \\
\hline Developed Interpersonal Skills & 0.728 & 0.019 & 39.19 & $47 \%$ \\
\hline Being committed to the job & 0.758 & 0.020 & 38.29 & $43 \%$ \\
\hline Knowing Self & 0.574 & 0.023 & 24.69 & $67 \%$ \\
\hline Clarity of beliefs and values & 0.461 & 0.032 & 14.44 & $79 \%$ \\
\hline \multicolumn{5}{|l|}{ THE CARE ENVIRONMENT } \\
\hline Skill mix & 0.569 & 0.026 & 21.63 & $68 \%$ \\
\hline Shared decision making systems & 0.762 & 0.019 & 39.64 & $42 \%$ \\
\hline Effective staff relationships & 0.622 & 0.025 & 24.96 & $61 \%$ \\
\hline Power sharing & 0.725 & 0.020 & 35.44 & $47 \%$ \\
\hline Potential for innovation and risk taking & 0.558 & 0.029 & 19.35 & $69 \%$ \\
\hline The Physical Environment & 0.634 & 0.026 & 24.60 & $60 \%$ \\
\hline Supportive Organisation Systems & 0.602 & 0.024 & 25.27 & $64 \%$ \\
\hline \multicolumn{5}{|l|}{ CARE PROCESSES } \\
\hline Working with patients beliefs and values & 0.796 & 0.017 & 47.32 & $37 \%$ \\
\hline Shared Decision making & 0.730 & 0.019 & 39.07 & $47 \%$ \\
\hline Engagement. & 0.677 & 0.022 & 30.42 & $54 \%$ \\
\hline Sympathetic Presence & 0.801 & 0.016 & 50.18 & $36 \%$ \\
\hline Providing holistic care & 0.770 & 0.016 & 49.42 & $41 \%$ \\
\hline
\end{tabular}

Table 5. Factor loading of first order factors to second order factors

\section{Relationship between Second Order Latent Variables}

Examination of the relationship between the second order latent factors show Prerequisites on Care Environment $\mathbf{0 . 7 2 9}$ ( $S E=0.027, E S T / S E=27.45, P=0.00)$; Prerequisites on Care Processes 0.875 ( $S E=0.023, E S T / S E 38.401, P=0.00)$. Care Environment on Care Processes 0.031 ( $S E=0.081$, EST/SE 0.45, $\mathrm{P}=0.07$ ). A standard unit change in Prerequisites will produce a 
0.685 unit change in Care Environment; a 1 unit change in Prerequisites will produce a .875 unit change in Care Processes (See Figure 2). There was no significant relationship between care environment and care process.

\section{Insert Figure 2 here.}

'Shared Decision-making' was the most significant predictor of 'Care Environment' and all seven constructs contributed significantly to Care Environment. All five constructs contributed significantly to 'Care Processes' but 'Sympathetic Presence' was the main contributor.

\section{Discussion}

Person-centred practice provides a central tenet underpinning health and social care internationally [1, 2]. The Person-centred Practice Framework [11] is internationally recognised and is being implemented globally [24]. This paper provides for the first time, statistical evidence of the relationships between the theoretical constructs of the framework and what these might mean for developing our understanding of person-centredness and its operationalisation in healthcare practice. Whilst the data reinforces the importance of the constructs of the framework and their relevance to person-centred healthcare, a number of issues are raised by the findings of the study.

The significance of interpersonal skills as a core component of person-centred practice is reinforced by the finding that the constructs 'Developed Interpersonal Skills' and 'Being committed to the job' offer the largest contribution to 'the 'Prerequisites' domain. The prerequisites of the Person-centred Practice Framework focus on the qualities of the practitioner that need to be in place in order for person-centred practice to be realised. The fact that interpersonal skills and commitment to the job emerge as the most significant qualities that need to be in place for person-centred practice to happen is an illuminating finding. In their writing, McCormack and McCance [10] argue that person-centredness is essentially a relational practice that is dependent on wellestablished interpersonal skills that can be operationalised in different practice contexts. The significance of interpersonal relationships also reinforces the idea that person-centredness is more than the 'doing' of particular practices, but is much more about a way of 'being' as a practitioner. The dominant focus in healthcare developments and innovations on doing personcentredness is one of the reasons why despite more than 10 years of quality improvements focusing on person-centred practices, we continue to see problems in practice and the existence of what Laird et al [25] have described as 'person-centred moments' at best. Commitment to the job further reinforces the being of person-centredness. McCance \& McCormack [11] define commitment to the job as 'the dedication of practitioners demonstrated to patients, families, and communities through intentional engagement that focuses on achieving the best possible outcomes'(pp55). Dedication implies presence and being in the moment with patients, families and communities and through such presence enables engagement. The findings of this study reinforce the need for practitioners to have well-developed interpersonal skills that will enable them to be present with patients and families.

The fact that 'clarity of values and beliefs' had the least significance in explaining the theory, raises important issues about how person-centred practice cultures are developed. The data shows that values and beliefs are important and of course are essential to the being of the person-centred practitioner. However, the data also suggests that having clear beliefs and values is not enough in itself for person-centred healthcare. In contemporary healthcare there is significant emphasis placed on values and beliefs among healthcare teams through programmes such as 'Values in Action' https://www.hse.ie/eng/about/our-healthservice/values-in-action/valuesinactionblog/how-the-nine-values-in-action-behaviours-were-developed.html that reinforce the importance of particular values being evident in healthcare practice behaviours. What such programmes fail to recognise, however, are the complex factors that have to be addressed on a continuous basis for such values to be translated into everyday practice. Emancipatory and transformational practice development [5] methodologies however have played a significant role in 
demonstrating the need for continuous facilitated meaningful engagement to develop person-centred health cultures. Evidence from practice development programmes show how clarity of beliefs and values acts as a foundation for culture change, leadership development, team effectiveness and consistency in patient care [26, 27]. Notably, such programmes also depend on long-term engagement for the development and embedding of culture change in healthcare settings - it is not a quick-fix solution. This is both a strength and a weakness of practice development as in a fast-moving healthcare context, quick-fix solutions that are the artefacts of person-centredness will always be favoured. Furthermore, the findings from this study suggest that this focus needs to be questioned and challenged if we are to see a large-scale shift towards person-centred cultures becoming a norm in healthcare organisations.

The data also suggests that the 'shared decision-making systems' construct of the care environment domain was the most likely predictor of a person-centred culture. This finding reinforces the importance of interdisciplinarity and service-user participation in healthcare practice. An organisational commitment to collaborative, inclusive and participative ways of engaging within and between teams is essential for person-centred practice [11] (McCormack \& McCance 2017). Shared decision-making among team members is the foundation of interdisciplinary practice [28] and the essence of person-centred healthcare. Ensuring that serviceusers play a key role in shaping their care plans is fundamental to person-centred practice and something that has been demonstrated to person-centred outcomes [29, 30]. Research by Ekman and colleagues shows that when patients are active agents in the development of a care plan, when healthcare teams collaborate to ensure the implementation of the plan and when evaluation of the impact of the plan is undertaken from the perspective of the patient, then patient and team outcomes can be demonstrated. The findings from the research reported in this paper reinforce the importance of this collaborative working and illustrate engagement in action and negotiation being achieved.

From the perspective of the 'person-centred processes', it is illuminating that the data suggests that being sympathetically present is core to all of the other person-centred processes - thus suggesting that being sympathetically present is a core person-centred practice. McCance \& McCormack [11] (pp.57) define being sympathetically present as - "An engagement that recognises the uniqueness and value of the patient by appropriately responding to cues that maximise coping resources through the recognition of important agendas in the person's life". The statistical significance of sympathetic presence in this research further highlights the need for the professional development of healthcare practitioners to focus as much on helping them develop their 'ways of being' as much as it does focus on the competence of what they do. Being sympathetically present is a phenomenological process that reinforces the individuality of persons. As Callaghan [31] (pp.21) asserts:

One of the difficulties in dealing with anything related to human experience is caused by no two people being exactly alike. There is so much that we have in common that we are inclined to imagine everyone is the same. Everybody is not. That which has meaning for one person may have none for another. Something of immense significance for one will mean little or nothing to another. The basic axiom is that each person is an individual and as such, a unique entity. This must never be forgotten in our attempts at self awareness or in our dealings with others"

Recognising this individuality is core to person-centred practice and the evidence from the research reported here reinforces that it is not something that person-centred practitioners do as a 'practice' but that it is an essence of being person-centred and underpins all practices. This is a significant finding from this research as it begins to surface issues that healthcare organisations may need to address for person-centred cultures to become a norm, including the reorganisation of services, challenging the standardisation agenda and protocolised care, as well as support systems for practitioners to enable them to sustain such individualised and engaged ways of being. 
The findings from the study provide new and innovative data relating to the realisation of person-centredness in healthcare and redress issues relating to the translation of person-centred principles into practice as identified by Moore et al [13] and Sharp and colleagues [14]. The evidence suggests that all the theoretical constructs make a statistical significant contribution to the overall understanding of person-centred practice and with varying degree of significance. Importantly, the findings highlight the focus on specific aspects of the framework to identify areas for change, facilitate change and evaluate it, so that the development of person-centred cultures can be sustained as a continuous focus in quality improvement programmes.

The findings add to a growing evidence base for a psychometrically sound tool [12, 19], that maps onto an established theoretical framework, redressing De Silva's [9] critique of tools to measure person-centred practice and relate the findings to an acceptable theoretical framework. The PCPI-S has shown its value in measuring person-centred practice [32]. The findings from this study continue to demonstrate that the PCPI-S has acceptable psychometric properties and displays the usefulness of the instrument as a means of informing how a theoretically driven approach to developing person-centredness in healthcare can be systematically developed through targeted interventions. The findings show the role that 'prerequisites' (practitioner qualities) play in shaping the 'care environment' and engaging in 'person-centred processes'. This is as expected in McCormack and McCance's [11] theoretical framework and indicates that a focus on 'prerequisites' can produce significant changes in shaping the overall approach to realising person-centred healthcare cultures.

\title{
Limitations
}

Only minor modifications (correlated errors) were required to the model to provide acceptable fit statistics. The presence of correlated errors indicates the presence of influential and as yet unmeasured elements. However, the Person-centred Practice Framework [11] contains elements (e.g. the macro context) that are not measured by the PCPI-S and should be included in modifications to the instrument. This would provide a comprehensive assessment of person-centred practice and further help with the translational process. The theoretical framework requires further testing with varying samples, to further explore its potential and psychometric properties.

\section{Conclusions}

Person-centred practice is an internationally recognised indicator of good practice and the Person-centred Practice Framework is a strong theoretical model for realising this in everyday practice. This paper provides statistical evidence to support the Personcentred Practice Framework and uses an instrument that appears to effectively measure the relationships between the constructs underpinning the theory. This is the first study that has attempted to articulate the relationship between person-centred constructs informed by an established theory. Ongoing research is needed to further test these relationships and build a reputable model of quality improvement that is theoretically driven and informs the development of targeted interventions that fulfil the need to be context-specific, but replicable across organisations and international contexts.

\section{Abbreviations}

\author{
$\mathrm{CFI}=$ Comparative Fit Index \\ DF $=$ Degrees of Freedom \\ Est $=$ Estimate \\ PCPF $=$ Person-centred Practice Framework \\ PCPI-S = Person-centred Practice Inventory - Staff \\ RMSEA $=$ Root Mean Square Error or Approximation \\ $\mathrm{SE}=$ Standard Error
}

Page 12/16 
TFI = Tucker Lewis Index

\section{Declarations}

\section{Ethical Approval}

Full ethical approval for the study was gained from the relevant institutions (Ulster University Nursing Filter Committee, Letterkenny University Hospital and Sligo University Hospital Ethics Committee). All ethical guidelines were adhered to during the study. Informed consent for use of the data was gained from all participants using implied consent. This ensured the anonymity of each submission. Participants received a participant information sheet explaining that the completion and return for the questionnaire implied consent to use the data.

\section{Consent for Publication}

Not applicable

\section{Availability of Data and Materials}

The datasets used and/or analysed during the current study are available from the corresponding author on reasonable request.

\section{Competing Interests}

The authors declare that they have no competing interests.

\section{Funding}

Non-applicable

\section{Author contributions}

BmC, TmC , PS designed the study and along with DmC collected the data.. PS analysed the data and DmC assisted with the analysis. BmC, TmC and PS wrote the paper and edited the paper. Final manuscript was agreed by all authors. All authors have read and approved the manuscript.

\section{Acknowledgements}

The authors would like to acknowledge all the health professionals across Northern and Southern Ireland for participating in the study. Also, thank you to the Health Service Executive Nursing and Midwifery Planning and Development Unit North West, Ireland for helping facilitate the work. 


\section{References}

1. World Health Organisaiton Global strategy on people-centred and integrated health services. 2015. WHO, Geneva.

2. Department of Health Health and Well-being 2026 - Delivering Together. 2016. DoH, Belfast.

3. McCormack, B \& McCance T Person-Centred Practice in Nursing and Healthcare: Theory and Practice. 2017. Oxford, WileyBlackwell.

4. Health Foundation Person-centred care made simple. 2014. London, The Health Foundation.

5. McCormack, B. Action research for the implementation of complex interventions, in DA Richards and I Rahm Hallberg, Complex Interventions in Health: an overview of research methods, 2015. Routledge, London.

6. Dewing J \& McCormack B Editorial: Tell me, how do you define person-centredness. Journal of Clinical Nursing. 2016; 26, 2509-2510.

7. Røsvik J, Kirkevold M, Engedal K, Brooker D, Kirkevold Ø. A model for using the VIPS framework for person-centred care for persons with dementia in nursing homes: a qualitative evaluative study. International Journal of older People Nursing. 2011; 6(3): 227-36.

8. Lynch BM, McCance T, McCormack B \& Brown D. The development of the Person-Centred Situational Leadership Framework: Revealing the being of person-centredness in nursing homes. Journal of Clinical Nursing, 2018; 27(1-2):427-440. doi: 10.1111/jocn.13949

9. DeSilva D. Helping Measure Person-centred Care: A Review of Evidence about Commonly Used Approaches and Tools Used to Help Measure Person-centred Care. 2014. London: The Health Foundation.

10. McCormack, B \& McCance T. Person-Centred Practice in Nursing and Healthcare: Theory and Practice. 2010. Oxford, WileyBlackwell.

11. McCormack, B \& McCance T. Person-Centred Practice in Nursing and Healthcare: Theory and Practice. 2017. Second Ed. Oxford, Wiley-Blackwell

12. Slater P, McCance T \& McCormack B. The development and testing of the Person-centred Practice Inventory - Staff (PCPI-S). International Journal of Quality in Healthcare. 2017; 1;29(4):541-547

13. Moore L, Britten N, Lydahl D, Naldemirci Ö, Elam M \& Wolf A Barriers and facilitators to the implementation of person-centred care in different healthcare contexts. Scandinavian Journal of Caring Sciences. 2017; 31: 662-673.

14. Sharp S, McAllister M \& Broadbent M. The tension between person-centred and task focused care in an acute surgical setting: A critical ethnography. Collegian. 2018; 25(1): (11-17).

15. McCormack, B., \& McCance, T. V. Development of a framework for person-centred nursing. Journal of advanced nursing. 2006: 56(5), 472-479.

16. Fawcett, J. Analysis and Evaluation of Conceptual Models of Nursing. 1995. FA Davis.

17. McCormack B. A conceptual framework for person-centred practice with older people. International Journal of Nursing Practice. 2003; 9:202-209

18. McCance, T.V. Caring in nursing practice: the development of a conceptual framework. Research and Theory for Nursing Practice: An International Journal , 2003; Vol.17, No.2, pp.101-116.

19. Bing-Jonsson, P. C., Slater, P., McCormack, B., \& Fagerström, L. Norwegian translation, cultural adaption and testing of the Person-centred Practice Inventory-Staff (PCPI-S). BMC health services research. 2018; 18(1), 555.

20. Wang, J. and Wang, X., Structural equation modelling: Applications using Mplus. 2019. John Wiley \& Sons

21. Kenny D Respecification of Latent Variable Models. 2011. http://davidakenny.net/cm/respec.htm. Accessed 05 April 2020

22. Byrne, B. M. Structural equation modeling with Mplus: Basic concepts, applications, and programming. 2013. routledge.

23. Hair, J. F., Anderson, R. E., Tatham, R. L., \& William, C. BlackMultivariate Data Analysis. 2010.

24. Harding, E., Wait, S. and Scrutton, J. The State of Play in Person-centred Care. 2015. The Health Foundation, London

25. Laird E, McCance T, Brendan McCormack \& Gribben B. Patients' experiences of in-hospital care when nursing staff were engaged in a practice development programme to promote person-centredness: A narrative analysis study. International

Page $14 / 16$ 
Journal of Nursing Studies. 2015: Vol 52, pp. 1454-1462

26. McCance T.V., Gribben B, McCormack B and Laird E Promoting person-centred practice within acute care: The impact of culture and context on a facilitated practice development programme. International Practice Development Journal, 2013; 3(1): manuscript 2.

27. McCormack, B., Dickson, C., Smith, T., Ford, H., Ludwig, S., Moyes, R., Lee, L., Adam, E., Paton, T., Lydon, B and Spiller, J. 'It's a nice place, a nice place to be'. The story of a practice development programme to further develop person-centred cultures in palliative and end-of-life care, International Practice Development Journal, 2018; Volume 8, Issue 1, Volume 8, Issue 1, Article 2 https://doi.org/10.19043/ipdj81.002

28. Dogba, M.J., Menear, M., Stacey, D., Brière, N. and Légaré, F. The Evolution of an Interprofessional Shared Decision-Making Research Program: Reflective Case Study of an Emerging Paradigm. International Journal of Integrated Care. 2016; 16(3): 4

29. Ekman I, Swedberg K, Taft C, Lindseth A, Norberg A, Brink E, Carlsson J, Dahlin-Ivanoff S, Johansson, IL, Kjellgren K, Lidén E, Öhlén J, Olsson, LE, Rosén H, Rydmark M and Sunnerhagen KS. Person-centered care - Ready for prime time, European Journal of Cardiovascular Nursing. 2011; 10: 248-251

30. Ekman I, Wolf A, Olsson LE, Taft C, Dudas K, Schaufelberger M and Swedberg K. Effects of person-centred care in patients with chronic heart failure: the PCC-HF study. European heart journal. 2012; 33:1112-9.

31. Callaghan, W.S. Good Grief. 1999. Colourpoint Books, Newtownards, Northern Ireland)

32. Slater, P., McCance, TV., McCormack, B. (Exploring person-centred practice within acute hospital settings. Int Pract Dev J 2015; 5: article 9. Special Edition Sept

\section{Figures}

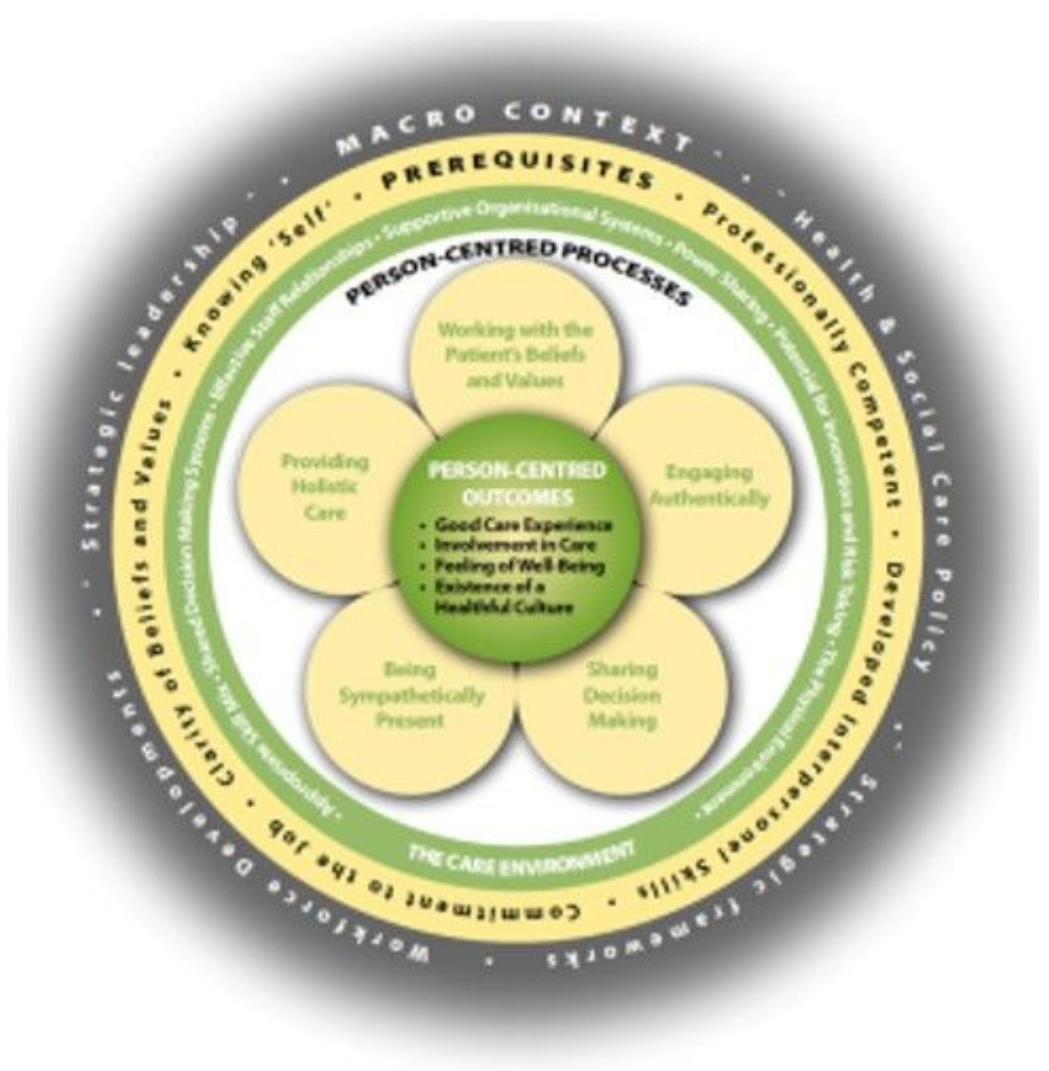

Figure 1

The Person-centred Practice Framework (McCormack and McCance 2010) 


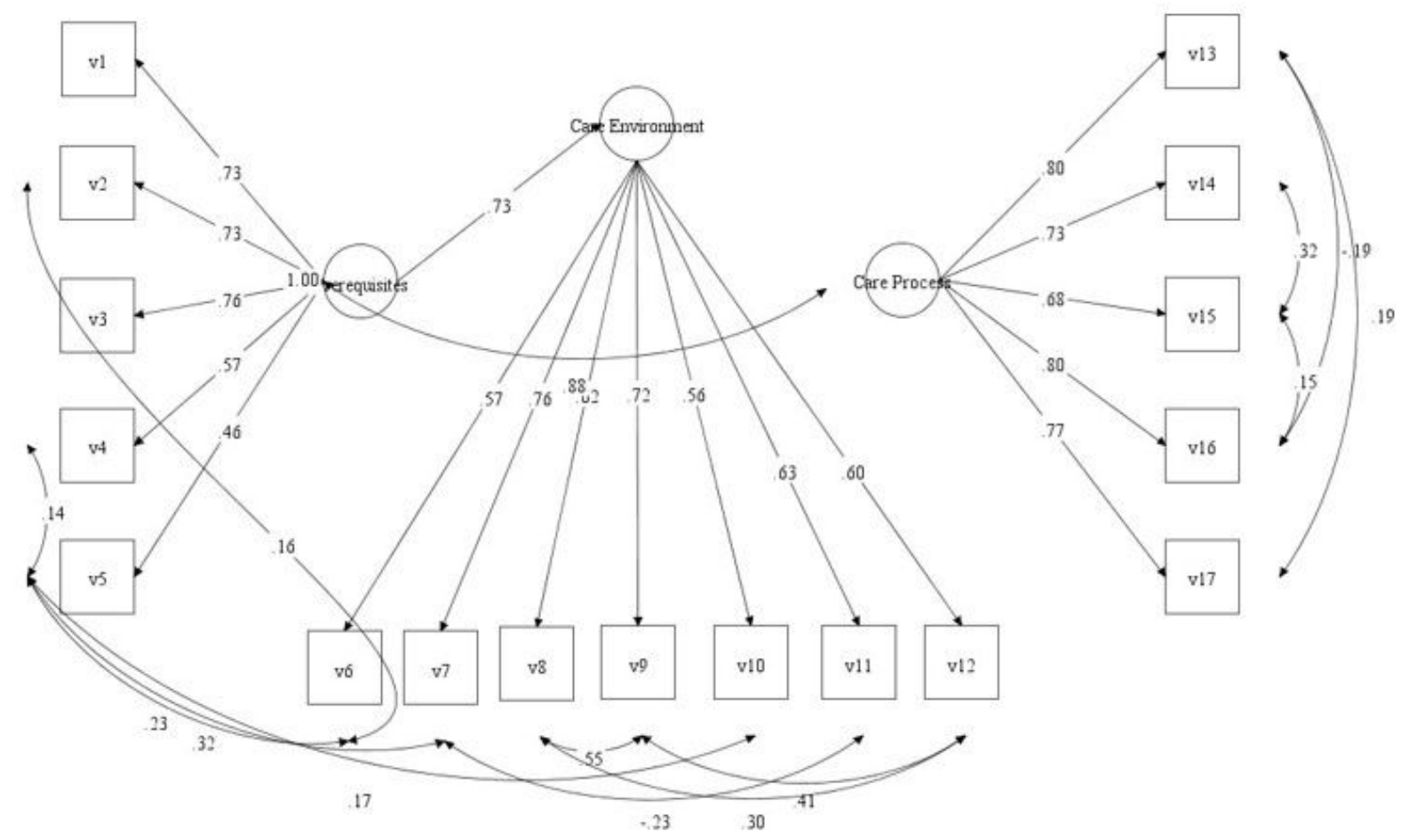

Figure 2

The Person-centred Practice Framework as a measurement model 